\title{
Tachykinin receptors antagonism for asthma: a systematic review
}

Renata Ramalho ${ }^{1,5^{*}}$, Raquel Soares ${ }^{2}$, Nuno Couto ${ }^{4}$ and André Moreira ${ }^{1,3}$

\begin{abstract}
Background: Tachykinins substance $P$, neurokinin A and neurokinin B seem to account for asthma pathophysiology by mediating neurogenic inflammation and several aspects of lung mechanics. These neuropeptides act mainly by their receptors NK1, NK2 and NK3, respectively which may be targets for new asthma therapy.

Methods: This review systematically examines randomized controlled trials evaluating the effect of tachykinins receptors antagonism on asthma. Symptoms, airway inflammation, lung function and airway inflammation were considered as outcomes. We searched the Cochrane Airways Group Specialized Register of Asthma Trials, Cochrane Database of Systematic Reviews, MEDLINE/PubMed and EMBASE. The search is as current as June 2010. Quality rating of included studies followed the Cochrane Collaboration and GRADE Profiler approaches. However, data were not pooled together due to different measures among the studies.
\end{abstract}

Results: Our systematic review showed the potential of NK receptor antagonist to decrease airway responsiveness and to improve lung function. However, effects on airway inflammation and asthma symptoms were poorly or not described.

Conclusion: The limited available evidence suggests that tachykinin receptors antagonists may decrease airway responsiveness and improve lung function in patients with asthma. Further large randomized trials are still required.

\section{Background}

A sharp increase in the prevalence, morbidity, mortality, and economic burden associated with asthma over the last 40 years, particularly in children is occurring. Approximately 300 million people worldwide have asthma, and its prevalence increases by $50 \%$ every decade [1]. Because no asthma definition exists, an operational definition was proposed by the Global Initiative for Asthma: a chronic inflammatory disorder of the airways associated with airway hyperesponsiveness that leads to recurrent episodes of wheezing, breathlessness, chest tightness, and coughing [1]. Therefore, asthma is a phenotypically heterogeneous disorder and, over the years, many different clinical subtypes of asthma have been described.

\footnotetext{
* Correspondence: rrenata@med.up.pt

'Department of Immunology, Faculty of Medicine, University of Porto, Porto, Portugal

Full list of author information is available at the end of the article
}

Recently, a model of interaction between different pathophysiologic mechanisms known to affect asthma phenotype was suggested [2]. This is of particular importance not only to recognize asthma as a complex disease for which different endogenous and exogenous factors may account, but also for emphasising the need of a precise definition of the asthma phenotype as a tool for improved asthma care. Despite major advances in understanding the pathogenesis of asthma and improvements in asthma drugs, the accompanying benefits have been less than expected. Drug strategies for asthma have been based on the premise that symptoms derive directly and immediately from airway inflammation focusing on the development of anti-inflammatory drugs, particularly steroids that show broad-spectrum inhibitory activity against a wide range of effector cells and their products. Evidence for an interaction between chronic inflammation and neural dysfunction points to an involvement linking the nervous and the immune system in the airways [3]. In this context, neuropeptides
Ciomed Central

() 2011 Ramalho et al; licensee BioMed Central Ltd. This is an Open Access article distributed under the terms of the Creative Commons Attribution License (http://creativecommons.org/licenses/by/2.0), which permits unrestricted use, distribution, and reproduction in any medium, provided the original work is properly cited. 
and neurotrophins have been recognized as key mediators of neuro-immune interactions [3] and investigation regarding the development of pharmacological compounds specifically targeting these molecules could be of interest in asthma.

Tachykinins include a family of neuropeptides with a wide range of actions in human body [4]. The most relevant are substance $\mathrm{P}$, neurokinin A (NKA) and neuroki$\operatorname{nin} \mathrm{B}(\mathrm{NKB})$ and act mainly by their receptors which are NK1, NK2 and NK3, respectively [5]. Interestingly, tachykinins are potent mediators of a number of functions in the airways [6]. Within human airways, substance $P$ and NKA are the predominant neuropeptides released from nonadrenergic-noncholinergic system by mechanical, thermal, chemical or inflammatory stimuli. NK3 receptors have been only recently considered in studies of airway regulation in health and in disease [7]. This is due to the observation that NKB, the most potent endogenous ligand for the NK3 receptor, is not readily localized to the airway nerves. In addition, contrasting the effects of NK1 and NK2 receptor activation in the airways, which induce pronounced and thus readily quantifiable effects in the lungs (e.g. bronchospasm, vasodilatation, vascular leakage, mucus secretion), the actions of NK3 receptor-selective agonists are mostly subtle and not simply measured with commonly used airway function measures [7].

Recent findings point to tachykinergic systems as promising targets of novel clinical agents. In asthma, the modulation of their receptors appear to influence a variety of pathological symptoms and processes such as inflammation [4]. However, improved therapeutic strategies can only be delineated if clinical effects are achieved by well-designed randomized controlled trials. Therefore, in an effort to assemble the clinical effects of tachykinin receptors modulation on asthmatic patients the authors conducted this systematic review.

\section{Methods}

\section{Studies, participants, interventions and outcomes}

The review was restricted to randomized controlled clinical trials and controlled trials, which studied the effect of tachykinin receptor antagonists on asthma related outcomes. Participants were adults or children with a medical diagnosis of asthma. All health-care settings were considered eligible and no restrictions for gender were made. The selected outcome measures were: symptoms, adverse events of the treatments, lung function, airway responsiveness and inflammation.

\section{Search strategy}

An initial search was carried out using the Cochrane Airways Group Specialized Register of asthma trials and EBM Reviews - Cochrane Database of Systematic
Reviews. Additional and separate searches were carried out on MEDLINE (1966-2010), EMBASE (1980-2010) and PubMed. The subject-specific search terms were "tac1r", "tac2r" "tac3r", "tachykinins", "tachykinins antagonists" "NK-1R”, "NK-2R”, "NK-3R", "substance P”, "neurokinin A", "neurokinin B", "asthma”. The reference lists of all primary studies were reviewed to identify trials not captured by electronic and manual searches. All titles and abstracts identified by the search that appeared relevant were selected for full text review. Update searches have been run up to June 2010.

\section{Data collection and analysis}

Abstracts of articles identified were viewed and articles that appear to fulfil the inclusion criteria were retrieved in full. Two reviewers (RR and NC) applied the inclusion criteria independently and where disagreement occurred they were resolved by consensus. Data were extracted by one reviewer (RR) and checked by other (AM). The methodological quality of the included trials was assessed with particular emphasis on allocation concealment and randomization, which was ranked using the Cochrane Collaboration and GRADE Profiler approaches [8]. This systematic review was in agreement with the PRISMA guidelines [9]. Meta-analysis was not conducted due to small number of included trials and heterogeneity across studies.

\section{Results}

Based on title, nineteen studies were identified as potentially relevant. However, based on abstract, only seven studies appeared to meet the inclusion criteria and their full text was obtained (Figure 1). Therefore, we included 7 studies comprising the use of five tachykinin receptors antagonists: NK-2 in two [10-12], NK-1/NK-2 in four [11,13-15] and NK-1/NK-2/NK-3 in other one [16], referring to a total of 98 subjects (Table 1 ). All studies used different doses of tachykinin receptors antagonists. Regarding treatment dosages, 5 studies used singledoses $[11-13,15,16]$ while in 2 studies doses of antagonists were given during 2 and 9 consecutive days $[10,14]$. Antagonists were administered by inhalation in 3 studies $[10,15,16]$ and orally in 4 studies [11-14]. None of the studies reported changes in asthma symptoms as an outcome.

Reviewer's judgments about risk of bias for each study was summarized in Figure 2. As shown in Figure 3, methodological quality of the included trials was graded as very low (Figure 2). Data extraction from included studies was summarized in Table 2.

\section{Airway responsiveness}

Five of the seven studies reported a decreased in airway responsiveness with the use of tachykinin receptors 


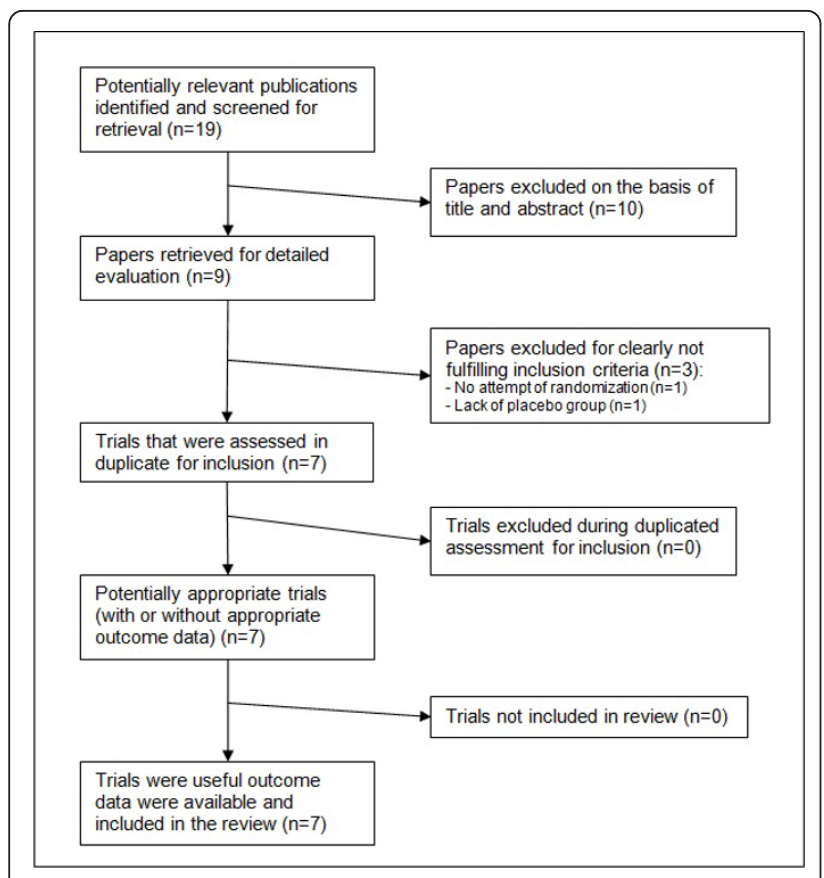

Figure 1 Flowchart of selection process. antagonists. However, this effect seemed to be dose dependent since in the study from Shelfhout the improvement of this outcome was not seen for the lowest dose tested [11]. These studies show, first, these drugs are indeed tachykinin receptors antagonists, and secondly, given the very modest shifts in the concentration response curves, nearly all of these drugs were underdosed.

\section{Lung Function}

Four studies reported lung function as an outcome. All but one failed to show significant effects with tachykinin receptors antagonists treatments [10,14-16]. However, in the study from Boot et al. [13] NK-1/NK-2 antagonist administration reverted the NKA-induced bronchoconstriction.

\section{Airway inflammation}

Only one study reported airway inflammation as an outcome [13]. In this study, authors concluded that airway inflammation evaluated either by changes in exhaled nitric oxide or sputum cells counts was not improved after a dual NK1/NK2 antagonist [13].

Table 1 Included studies on the effect of tachykinin receptors antagonism on asthma

\begin{tabular}{|c|c|c|c|c|}
\hline $\begin{array}{l}\text { Study/ } \\
\text { reference }\end{array}$ & $\begin{array}{l}\text { Design and } \\
\text { participants }\end{array}$ & Intervention & Outcomes & Results \\
\hline $\begin{array}{l}\text { Shelfhout et } \\
\text { al., } 2009 \text { [11] }\end{array}$ & $\begin{array}{l}\text { - Single-center, RCT, } \\
\text { double-blind, crossover } \\
\text { - } 12 \text { non-smoking } \\
\text { subjects males }\end{array}$ & $\begin{array}{l}\text { - } 2 \text { or } 8 \text { mg of MEN } 48968 \\
\text { (dual NK-1/NK-2 receptor } \\
\text { antagonist) single inhaled } \\
\text { dose }\end{array}$ & $\begin{array}{l}\text { - Airway responsiveness } \\
\text { to NK antagonist }\end{array}$ & - Decreased airway responsiveness \\
\hline $\begin{array}{l}\text { Boot et al., } \\
2007 \text { [13] }\end{array}$ & $\begin{array}{l}\text { - Single-center, RCT, } \\
\text { double-blind, crossover } \\
\text { - } 19 \text { non-smoking } \\
\text { subjects ( } 15 \text { females); } \\
\text { mean age } 30 \mathrm{yr}\end{array}$ & $\begin{array}{l}\text { - } 4.8 \text { mg of AVE } 5883 \text { (dual } \\
\text { NK-1/NK-2 receptor } \\
\text { antagonist) single inhaled } \\
\text { dose }\end{array}$ & $\begin{array}{l}\text { - Airway responsiveness } \\
\text { to NK antagonist } \\
\text { - Lung function } \\
\text { - Airway inflammation }\end{array}$ & $\begin{array}{l}\text { - Decreased airway responsiveness } \\
\text { - Inhibition of bronchoconstriction }\end{array}$ \\
\hline $\begin{array}{l}\text { Schelfhout et } \\
\text { al., } 2006 \text { [16] }\end{array}$ & $\begin{array}{l}\text { - Multi- center, RCT, } \\
\text { double-blind, crossover } \\
\text { - } 15 \text { subjects ( } 7 \text { females); } \\
\text { mean age } 30 \mathrm{yr}\end{array}$ & $\begin{array}{l}\text { - } 200 \text { mg CS-003 (triple NK-1/ } \\
\text { NK-2/NK-3 receptor } \\
\text { antagonist) single oral dose. }\end{array}$ & $\begin{array}{l}\text { - Airway responsiveness } \\
\text { to NK antagonist } \\
\text { - Lung function }\end{array}$ & - Decreased airway responsiveness \\
\hline $\begin{array}{l}\text { Joos et al., } \\
2004 \text { [15] }\end{array}$ & $\begin{array}{l}\text { - Multi- center, RCT, } \\
\text { double-blind, crossover } \\
\text { - } 18 \text { males; mean age } \\
28 \mathrm{yr}\end{array}$ & $\begin{array}{l}\text { - } 100 \text { mg DNK } 333 \text { (dual NK-1/ } \\
\text { NK-2 receptor antagonist) } \\
\text { single orally dose. }\end{array}$ & $\begin{array}{l}\text { - Airway responsiveness } \\
\text { to NK antagonist } \\
\text { - Lung function }\end{array}$ & - Decreased airway responsiveness \\
\hline $\begin{array}{l}\text { Kraan et al., } \\
2001 \text { [10] }\end{array}$ & $\begin{array}{l}\text { - Single-center, RCT, } \\
\text { double-blind, crossover } \\
\text { - } 12 \text { atopic non-smoking } \\
\text { males; mean age } 30 \mathrm{yr}\end{array}$ & $\begin{array}{l}\text { - } 100 \text { mg }(4 \times 25 \text { mg) SR } \\
\text { 48968C (NK-2 receptor } \\
\text { antagonist) orally once-daily } \\
\text { during } 9 \text { days. }\end{array}$ & $\begin{array}{l}\text { - Airway responsiveness } \\
\text { to NK antagonist } \\
\text { - Lung function }\end{array}$ & - No effect \\
\hline $\begin{array}{l}\text { Schoor et } \\
\text { al.,1998 [12] }\end{array}$ & $\begin{array}{l}\text { - Single-center, RCT, } \\
\text { double-blind, crossover } \\
\text { - } 12 \text { non-smoking males; } \\
\text { mean age } 29 \mathrm{yr} \text {. }\end{array}$ & $\begin{array}{l}\text { - } 100 \text { mg SR } 48968 \text { (NK-2 } \\
\text { receptor antagonist) orally } \\
\text { single dose }\end{array}$ & $\begin{array}{l}\text { - Airway responsiveness } \\
\text { to NK antagonist }\end{array}$ & - Decreased airway responsiveness \\
\hline $\begin{array}{l}\text { Joos et al., } \\
1996[14]\end{array}$ & $\begin{array}{l}\text { - Single-center, RCT, } \\
\text { double-blind, crossover } \\
\text { - } 10 \text { atopic non-smoking } \\
\text { subjects ( } 2 \text { females); } \\
\text { mean age } 27 \mathrm{yr}\end{array}$ & $\begin{array}{l}\text { - } 4 \text { mg FK224 (dual NK-1/NK-2 } \\
\text { receptor antagonist) inhaled } \\
\text { once-daily for } 2 \text { days. }\end{array}$ & $\begin{array}{l}\text { - Airway responsiveness } \\
\text { to NK antagonist } \\
\text { - Lung function }\end{array}$ & - No effect \\
\hline Summary & $\begin{array}{l}\text { - } 98 \text { young adults ( } 24 \\
\text { females, } 74 \text { males) }\end{array}$ & $\begin{array}{l}\text { - Seven different antagonists } \\
\text { acting on NK-1R, NK-2, NK-3 in } \\
\text { mono, dual or triple } \\
\text { combinations. }\end{array}$ & $\begin{array}{l}\text { - Airway responsiveness } \\
\text { to NK antagonist, lung } \\
\text { function and airway } \\
\text { inflammation. }\end{array}$ & $\begin{array}{l}\text { - Five studies showed positive effects on } \\
\text { airway responsiveness and/or lung function } \\
\text { and two studies failed to demonstrate effects } \\
\text { on asthma outcomes. }\end{array}$ \\
\hline
\end{tabular}




\begin{tabular}{|c|c|c|c|c|c|c|c|}
\hline & 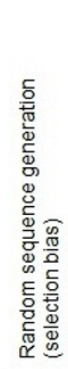 & 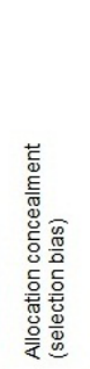 & 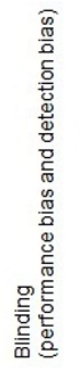 & 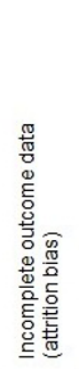 & 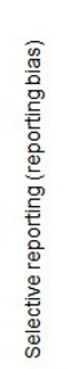 & 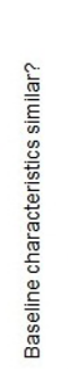 & 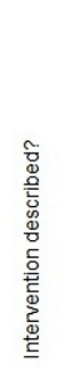 \\
\hline Shelfhout et al., 2009 & $\odot$ & (?) & $\oplus$ & $\odot$ & $\oplus$ & $\oplus$ & $\oplus$ \\
\hline Boot et al., 2007 & $\odot$ & (?) & $\odot$ & (?) & $\odot$ & $\odot$ & $\odot$ \\
\hline Schelfhout et al., 2006 & $\odot$ & (?) & $\odot$ & (?) & $\odot$ & $\odot$ & $\odot$ \\
\hline Joos et al., 2004 & $\odot$ & (?) & $\oplus$ & $\oplus$ & $\oplus$ & $\oplus$ & $\oplus$ \\
\hline Kraan et al., 2001 & $\odot$ & (?) & $\oplus$ & (?) & $\oplus$ & $\oplus$ & $\oplus$ \\
\hline Schoor et al.,1998 & $\odot$ & (?) & $\oplus$ & (?) & ๑ & $\oplus$ & $\oplus$ \\
\hline Joos et al., 1996 & $\odot$ & (?) & $\odot$ & (?) & $\oplus$ & $\odot$ & $\oplus$ \\
\hline High Risk ? & & רclear & ๑ & \multicolumn{4}{|c|}{ Low Risk } \\
\hline \multicolumn{8}{|c|}{$\begin{array}{l}\text { Figure } 2 \text { "Risk bias" summary: reviewer author's judgements } \\
\text { about each risk of bias item for included studies. }\end{array}$} \\
\hline
\end{tabular}

\section{Adverse events}

Adverse events were only considered as an outcome in three studies $[11,13,15]$. In the study from Boot et al. [13] adverse events were reported as transient self-limiting bronchospasm starting within 12 minutes after drug inhalation (8 patients under NK-1/NK-2 antagonist and 4 patients under placebo), headache ( 5 patients under NK-1/NK-2 antagonist and 3 patients under placebo), and self-limiting dyspneia (2 patients under NK-1/NK-2 antagonist and 5 patients under placebo). Shelfhout et al. [11] reported no serious adverse effect with the NK1/NK-2 antagonist studied. In the study from Joos et al. [15] was reported that 4 patients referred fatigue, headache, aggravated asthma, cough and wheezing. However, as no changes in laboratory parameters were observed authors concluded that these events were not related to the NK-1/NK-2 antagonist tested.

\section{Discussion}

Our systematic review showed: first, the use of NK receptor antagonist decreases airway responsiveness and may improve lung function; secondly effects on airway inflammation and asthma symptoms have been poorly or not addressed at all. It must be noted that these conclusions are limited by different interventions, doses,

Question: Should tachykinin receptors antagonists vs placebo be used for asthma?

Settings: Developed countries, outpatients

Bibliography:

\begin{tabular}{|c|c|c|c|c|c|c|c|c|c|c|c|c|}
\hline \multirow{2}{*}{\multicolumn{7}{|c|}{ Quality assessment }} & \multicolumn{5}{|c|}{ Summary of findings } & \multirow[b]{3}{*}{ Importance } \\
\hline & & & & & & & \multicolumn{2}{|c|}{ No of patients } & \multicolumn{2}{|c|}{ Effect } & \multirow[b]{2}{*}{ Quality } & \\
\hline $\begin{array}{c}\text { No of } \\
\text { studies }\end{array}$ & Design & Limitations & Inconsistency & Indirectness & Imprecision & $\begin{array}{c}\text { Other } \\
\text { considerations }\end{array}$ & $\begin{array}{l}\text { tachykinin } \\
\text { receptors } \\
\text { antagonists }\end{array}$ & placebo & $\begin{array}{l}\text { Relative } \\
(95 \% \mathrm{CI})\end{array}$ & Absolute & & \\
\hline \multicolumn{13}{|c|}{ Airway responsiveness (Better indicated by lower values) } \\
\hline 7 & $\begin{array}{l}\text { randomised } \\
\text { trials }\end{array}$ & $\begin{array}{l}\text { very } \\
\text { serious } 1,2,3,4\end{array}$ & serious $^{1,5}$ & $\begin{array}{l}\text { no serious } \\
\text { indirectness }\end{array}$ & $\begin{array}{l}\text { no serious } \\
\text { imprecision }\end{array}$ & none & 98 & 92 & - & $\begin{array}{c}\text { not } \\
\text { pooled }\end{array}$ & $\begin{array}{l}\text { ๑OOO } \\
\text { VERY } \\
\text { LOW }\end{array}$ & $\mathrm{CRITICAL}^{6,7}$ \\
\hline \multicolumn{13}{|c|}{ Lung function (Better indicated by higher values) } \\
\hline 5 & $\begin{array}{l}\text { randomised } \\
\text { trials }\end{array}$ & $\begin{array}{l}\text { very } \\
\text { serious }\end{array}$ & serious $^{1,3,5}$ & $\begin{array}{l}\text { no serious } \\
\text { indirectness }\end{array}$ & $\begin{array}{l}\text { no serious } \\
\text { imprecision }\end{array}$ & none & 74 & 68 & - & $\begin{array}{c}\text { not } \\
\text { pooled }\end{array}$ & $\begin{array}{l}\text { @OOO } \\
\text { VERY } \\
\text { LOW } \\
\end{array}$ & CRITICAL $^{8}$ \\
\hline \multicolumn{13}{|c|}{ Aiway inflammation (Better indicated by lower values) } \\
\hline 1 & $\begin{array}{l}\text { randomised } \\
\text { trials }\end{array}$ & $\begin{array}{l}\text { very } \\
\text { serious } \\
3,9\end{array}$ & very serious ${ }^{10}$ & $\begin{array}{l}\text { no serious } \\
\text { indirectness }\end{array}$ & \begin{tabular}{|l} 
no serious \\
imprecision
\end{tabular} & none & 3 & 3 & - & $\begin{array}{c}\text { not } \\
\text { pooled }\end{array}$ & $\begin{array}{l}\oplus O O O \\
\text { VERY } \\
\text { LOW }\end{array}$ & CRITICAL \\
\hline
\end{tabular}

1 Studies used four different antagonists acting on different receptors.

${ }^{2}$ Airway inflammation was assessed by using different bronchoconstrictors: methacholine, NKA, AMP.

${ }^{3}$ Lack of allocation concealment explanation.

${ }^{4}$ Major differences in dose and route of administration.

5 Major differences in interventions.

${ }^{6}$ Airway hyperresponsiveness is a characteristic feature of asthma and consists of an increased sensitivity of the airways to an inhaled constrictor agonists.

${ }^{7}$ Measurements of airway responsiveness are useful in making a disgnosis of asthma.

${ }^{8}$ Measurements of lung function are useful in making a disgnosis of asthma.

${ }^{9}$ Number of patients was too small.

${ }^{10}$ Only one study reported this outcome.

Figure 3 Tachykinin receptors antagonists compared to placebo for asthma grading evidence. 
Table 2 Effect of tachykinin receptors antagonism on asthma outcomes

\begin{tabular}{|c|c|c|c|c|c|}
\hline \multirow[b]{2}{*}{ Outcome } & \multirow[b]{2}{*}{$\mathbf{N}$} & \multicolumn{2}{|l|}{ Control } & Treated & \multirow[b]{2}{*}{$p$} \\
\hline & & Mean(SD) & $\mathbf{N}$ & Mean(SD) & \\
\hline \multicolumn{6}{|l|}{ - AIRWAY RESPONSIVENESS: } \\
\hline \multicolumn{6}{|l|}{$\log \mathrm{PC}_{35} \mathrm{sGaw}$ NKA mol/ml } \\
\hline FK224 (NK-1R/NK-2R antagonist) & 10 & $-6.04(0.18)$ & 10 & $-6.19(0.23)$ & n.s. \\
\hline \multicolumn{6}{|l|}{ Joos et al, 1996} \\
\hline \multicolumn{6}{|l|}{$\mathrm{PC}_{20}-\mathrm{AMP} \mathrm{mg} / \mathrm{ml}$} \\
\hline \multicolumn{6}{|l|}{ SR 48968C (NK-1R/NK2R antagonist) } \\
\hline Kraan et al, 2001 & 12 & $1.46(1.11)$ & 6 & $1.25(0.98)$ & n.s. \\
\hline \multicolumn{6}{|l|}{$\log _{10} \mathrm{PC}_{20}-\mathrm{NKA} \mathrm{mol} / \mathrm{ml}$} \\
\hline \multicolumn{6}{|l|}{ MEN 11420 (NK-2R antagonist) } \\
\hline Shelfhout et al, 2009 & 12 & $-6.38(0.26)$ & 12 & $-6.92(0.25)$ & 0.028 \\
\hline \multicolumn{6}{|l|}{ AVE 5883 (NK-1R/NK-2R antagonist) } \\
\hline Boot et al, 2007 & 19 & $-6.40(0.12)$ & 19 & $-6.74(0.12)$ & 0.004 \\
\hline \multicolumn{6}{|c|}{ CS-003 (NK-1R/NK-2R/NK-3R antagonist) } \\
\hline Schelfhout et al, 2006 & 15 & $-5.92(0.83)$ & 15 & $-6.99(0.66)$ & $<0.05$ \\
\hline \multicolumn{6}{|l|}{ DNK 333 (NK-1R/NK-2R antagonist) } \\
\hline Joos et al, 2004 & 18 & $-5.60^{*}$ & 18 & $-6.8^{*}$ & $<0.001$ \\
\hline \multicolumn{6}{|l|}{ SR 48068 (NK-2R antagonist) } \\
\hline Schoor et al, 1998 & 12 & $-7.02(0.28)$ & 12 & $-7.64(0.19)$ & 0.05 \\
\hline \multicolumn{6}{|l|}{ - LUNG FUNCTION: } \\
\hline \multicolumn{6}{|l|}{$\mathrm{FEV}_{1}$ (\% fall) } \\
\hline \multicolumn{6}{|l|}{ AVE 5883 (NK-1R/NK-2R antagonist) } \\
\hline Boot e tal, 2007 & 19 & $145.5(11.7)$ & 19 & $116.2(11.7)$ & 0.01 \\
\hline \multicolumn{6}{|l|}{ SR 48968C (NK-1R/NK2R antagonist) } \\
\hline Kraan e tal, 2001 & 12 & $81(4)$ & 6 & 78(3) & n.s. \\
\hline \multicolumn{6}{|c|}{$\mathrm{FEV}_{1}(\mathrm{~L})$} \\
\hline \multicolumn{6}{|c|}{ CS-003 (NK-1R/NK-2R/NK-3R antagonist) } \\
\hline Schelfhout e tal, 2006 & 15 & $3.34(0.62)$ & 15 & $3.25(0.71)$ & n.s. \\
\hline \multicolumn{6}{|l|}{ FK224 (NK-1R/NK-2R antagonist) } \\
\hline Joos e tal, 1996 & 10 & $3.66 \times 10^{-3}(0.25)$ & 10 & $3.67 \times 10^{-3}(0.21)$ & n.s. \\
\hline \multicolumn{6}{|l|}{ - AIRWAY INFLAMMATION: } \\
\hline \multicolumn{6}{|l|}{ Eosinophils \% } \\
\hline \multicolumn{6}{|l|}{ AVE 5883 (NK-1R/NK-2R antagonist) } \\
\hline Boot e tal, 2007 & 19 & $4.86(1.75)$ & 19 & $3.33(1.58)$ & n.s. \\
\hline \multicolumn{6}{|l|}{ eNO ppb } \\
\hline AVE 5883 (NK-1R/NK-2R antagoni & & & & & \\
\hline Boot e tal, 2007 & 19 & $37.64(6.40)$ & 19 & $43.44(6.57)$ & 0.32 \\
\hline
\end{tabular}

* 95\% Confidence Interval for the difference in $\log _{10} \mathrm{PC}_{20}-\mathrm{NK}, 0.841-1.616$.

$\mathrm{SP}$ - substance P; eNO - exhaled nitric oxide; n.s. - reported as not significant.

route of administration, schedules and samplings, as well as patient characteristics. Nevertheless, our review was strengthened by the use of a complete search strategy and the omission of relevant studies seems unlikely.

The most important limitation of our review was the relatively small number of well-designed, adequately powered studies reporting all outcomes of interest. Additional limitations were: each of seven studies used a different drug, most of these studies failed to document adequate dosing, and nearly every study targeted a different combination of the NK1, NK2 and NK3.
The effectiveness of tachykinin receptors antagonism on cough and chest tightness was not reported. Moreover, airway inflammation was only discussed in one study. As an inflammatory condition, evaluation of the effect of drugs in airway inflammation is a major outcome in asthma. Few studies analysed drug safety and reported side effects. However, the undesired effects observed seemed to be not related to the use of antagonists. Synthesis of the literature with such few studies was difficult because a single study may affect the outcomes. In this situation, heterogeneity may be 
introduced and publication bias may be not easy to detect.

Selective tachykinin receptors antagonists tested on guinea pigs have been shown to inhibit the late allergic and airway hyperesponsiveness and reduce eosinophilic infiltration and vascular permeability $[8,17,18]$. These models showed promising effects of dual and triple antagonists in reducing cough and vascular hyperpermeability and inhibiting airway hyperesponsiveness and bronchoconstriction. Similar results were observed in studies using ferrets and mice providing additional evidence in reducing mucus production $[3,19]$. However, as for other studies involving animals, the extrapolation for humans has been difficult.

Playing substance $\mathrm{P}$ the more relevant role in allergeninduced airway inflammation, the lack of effectiveness in reducing airway hyperesponsiveness may be due to compromising of results by testing antagonists' protection against NKA inhalation. It is recognized that substance $\mathrm{P}$, acting through its NK-1 receptor, induces bronchoconstriction and airway hyperesponsiveness, with asthmatics being more sensitive $[5,15]$. Acting through its NK-2 receptor, inhaled NKA had been shown to be more responsible for cause bronchoconstriction [16]. Lack of efficacy in other studies may also be explained by requirement of different concentrations of antagonists to inhibit bronchoconstriction.

The use of tachykinin receptors antagonists in dual or triple combination rather than one antagonist alone may be regard as a more efficient way of improve asthma outcomes. However, the use of these antagonists in combination does not allow us to determine the relative contribution of each tachykinin receptor subtype to bronchoconstrictor effect of NKA and SP in asthma. In fact, these receptors have very different effects on airway cells (Table 3), airway vascular smooth muscle and central nervous system reflexes. Future investigation should compare the use of NK-1 and NK-2 receptors antagonists alone and in combination in order to set the relative contribution of NKA and SP antagonism in asthma. Furthermore, it seems imperative to gain more insight into the role of

Table 3 Effects of tachykinins receptors NK1, NK2 AND NK3 on human airway cells

\begin{tabular}{cccc}
\hline & \multicolumn{3}{c}{ Receptors } \\
\hline Effect on human airway cells & NK1 & NK2 & NK3 \\
\hline Chemotaxis & +++ & - & - \\
Lymphocyte proliferation & +++ & + & - \\
Increased neutrophil motility & +++ & + & - \\
Monocyte/macrophage stimulation & ++ & + & - \\
Mast cell activation & ++ & + & - \\
\hline
\end{tabular}

Modified from Joos et al. [17] tachykinin receptors pathways as well as into the effectiveness of their antagonisms on asthma related symptoms like cough and chest tightness.

\section{Conclusions}

Overall we conclude that the number of studies on tachykinin receptors modulation in asthma is very small and limited both in sample size and patients characteristics, as well as in asthma outcome results. However, evidence on the role of tachykinin receptors antagonism in asthma has been gathered and their potential to regulate some airway functions has been recognized. Longer randomized trials standardized rating scales and outcome measures, at similar time periods and with similar antagonists are required. This further research will also be important to allow investigators to further define the role of SP and NKA in asthma pathogenesis.

\section{Acknowledgements}

We would like to thank to Professor Guy F. Joos for providing articles on request. We thank the reviewers for their comments that helped us to improve the manuscript. We declare that we had no financial supporting

\section{Author details}

${ }^{1}$ Department of Immunology, Faculty of Medicine, University of Porto, Porto, Portugal. ${ }^{2}$ Department of Biochemistry, Faculty of Medicine, University of Porto, Porto, Portugal. ${ }^{3}$ Department of Immunoallergology, Hospital of São João E.P.E., Porto, Portugal. ${ }^{4}$ Portuguese Oncology Institute IPOPFG-E.P.E, Porto, Portugal. ${ }^{5}$ Centro de Dermatologia Epidermis, Instituto CUF, Porto, Portugal.

\section{Authors' contributions}

RR and AM designed the protocol. RR and NC performed the searches. RR performed the selection and data extraction. AM checked the data. RR drafted the manuscript. AM and RS revised the manuscript. All authors read and approved the final manuscript.

\section{Competing interests}

The authors declare that they have no competing interests.

Received: 25 August 2010 Accepted: 2 August 2011

Published: 2 August 2011

\section{References}

1. GINA: Global Strategy for Asthma Management and Prevention. Global Initiative for Asthma (GINA) ed 2008.

2. Anderson GP: Endotyping asthma: new insights into key pathogenic mechanisms in a complex, heterogeneous disease. Lancet 2008, 372(9643):1107-19.

3. Veres TZ, Rochlitzer $\mathrm{S}$, Braun A: The role of neuro-immune cross-talk in the regulation of inflammation and remodelling in asthma. Pharmacol Ther 2009, 122(2):203-14.

4. Satake $H$, Kawada T: Overview of the primary structure, tissuedistribution, and functions of tachykinins and their receptors. Current Drug Targets 2006, 7(8):963-74.

5. Devane $\mathrm{CL}$, Substance P: A new era, a new role. Pharmacotherapy 2001 21(9l):1061-9.

6. Groneberg DA, Harrison S, Dinh QT, Geppetti P, Fischer A: Tachykinins in the respiratory tract. Current Drug Targets 2006, 7(8):1005-10.

7. Canning B: Neurokinin3 receptor regulation of the airways. Vascular Pharmacology 2006, 45:227-34.

8. Balshem $H$, Helfand M, Schünemann HJ, Oxman AD, Kunz R, Brozek J, et al: GRADE guidelines: 3 . Rating the quality of evidence. Journal of Clinical Epidemiology 2011, 64(4):401-6. 
9. Moher D, Liberati A, Tetzlaff J, Altman D: Preferred Reporting Items for Systematic Reviews and Meta-Analysis: The PRISMA Statement. PloS Med 2009, 6(6):e1000097.

10. Kraan J, Vink-Klooster H, Postma DS: The NK-2 receptor antagonist SR 48968C does not improve adenosine hyperresponsiveness and airway obstruction in allergic asthma. Clin Exp Allergy 2001, 31(2):274-8.

11. Schelfhout V, Van De Velde V, Maggi C, Pauwels R, Joos G: The effect of the tachykinin NK(2) receptor antagonist MEN11420 (nepadutant) on neurokinin A-induced bronchoconstriction in asthmatics. Ther Adv Respir Dis 2009, 3(5):219-26

12. Van Schoor J, Joos GF, Chasson BL, Brouard RJ, Pauwels RA: The effect of the NK2 tachykinin receptor antagonist SR 48968 (saredutant) on neurokinin A-induced bronchoconstriction in asthmatics. Eur Respir J 1998, 12(1):17-23.

13. Boot JD, de Haas S, Tarasevych S, Roy C, Wang L, Amin D, et al: Effect of an NK1/NK2 receptor antagonist on airway responses and inflammation to allergen in asthma. Am J Respir Crit Care Med 2007, 175(5):450-7.

14. Joos GF, Schoor J, Kips JC, Pauwels RA: The effect of inhaled FK224, a tachykinin NK-1 and NK-2 receptor antagonist, on neurokinin A-induced bronchoconstriction in asthmatics. Am J Respir Crit Care Med 1996, 153(6 Pt 1):1781-4

15. Joos G, Vincken W, Louis R, Schelfhout VJ, Wang JH, Shaw MJ, Cioppa GD, Pauwels RA: Dual NK1/NK2 antagonist DNK333 inhibits neurokinin Ainduced bronchonstriction in asthma patients. Eur Respir J 2004, 23:76-81.

16. Schelfhout $V$, Louis $R$, Lenz W, Heyrman R, Pauwels $R$, Joos G: The triple neurokinin-receptor antagonist CS-003 inhibits neurokinin A-induced bronchoconstriction in patients with asthma. Pulm Pharmacol Ther 2006, 19(6):413-8.

17. Joos GF, Pauwels RA: Tachykinin receptor antagonists: potential in airways diseases. Current Opinion in Pharmacology 2001, 1(3):235-41.

18. Schuiling $M$, Zuidhof $A B$, Zaagsma J, Meurs $H$ : Involvement of tachykinin NK1 receptor in the development of allergen-induced airway hyperreactivity and airway inflammation in conscious, unrestrained guinea pigs. Am J Respir Crit Care Med 1999, 159(2):423-30.

19. Khawaja AM, Liu Y, Rogers DF: Effect of non-peptide tachykinin NK(1) receptor antagonists on non-adrenergic, non-cholinergic neurogenic mucus secretion in ferret trachea. Eur J Pharmacol 1999, 384(2-3):173-81.

\section{Pre-publication history}

The pre-publication history for this paper can be accessed here: http://www.biomedcentral.com/1471-2466/11/41/prepub

doi:10.1186/1471-2466-11-41

Cite this article as: Ramalho et al.: Tachykinin receptors antagonism for asthma: a systematic review. BMC Pulmonary Medicine 2011 11:41.

\section{Submit your next manuscript to BioMed Central and take full advantage of:}

- Convenient online submission

- Thorough peer review

- No space constraints or color figure charges

- Immediate publication on acceptance

- Inclusion in PubMed, CAS, Scopus and Google Scholar

- Research which is freely available for redistribution 